\title{
Medulla Oblongata
}

National Cancer Institute

\section{Source}

National Cancer Institute. Medulla Oblongata. NCI Thesaurus. Code C12442.

The lower portion of the brainstem located between the pons and brainstem. This structure contains several descending and ascending tracts, lower cranial nerve nuclei, a significant proportion of the reticular system of the brainstem and other structures. 